\title{
An Uncertain Data Integration System
}

\author{
Naser Ayat ${ }^{\# 1}$, Hamideh Afsarmanesh\#2, Reza Akbarinia ${ }^{* 3}$, Patrick Valduriez ${ }^{* 4}$ \\ \# Informatics Institute, University of Amsterdam, Amsterdam, Netherlands \\ ${ }^{1}$ s.n.ayat@uva.nl, ${ }^{2}$ h.afsarmanesh@uva.nl \\ *INRIA and LIRMM, Montpellier, France \\ ${ }^{3,4}\{$ Firstname.Lastname@inria.fr\}
}

\begin{abstract}
Data integration systems offer uniform access to a set of autonomous and heterogeneous data sources. An important task in setting up a data integration system is to match the attributes of the source schemas. In this paper, we propose a data integration system which uses the knowledge implied within functional dependencies for matching the source schemas. We build our system on a probabilistic data model to capture the uncertainty arising during the matching process. Our performance validation confirms the importance of functional dependencies and also using a probabilistic data model in improving the quality of schema matching. Our experimental results show significant performance gain compared to the baseline approaches. They also show that our system scales well.
\end{abstract}

Keywords: data integration, schema matching, uncertain data integration, functional dependency

\section{Introduction}

The ultimate goal of data integration systems is to provide a uniform query interface to multiple heterogeneous and autonomous data sources. Sources may reside within an enterprise or on the web, and their numbers can range from tens to thousands. The main building blocks of a typical data integration application are mediated schema definition, schema matching and schema mapping.

The mediated schema is the schema on which users pose queries. Schema matching is the process of finding associations between the elements (often attributes or relations) of different schemas, e.g. a source schema and the mediated schema in the popular Local As View (LAV) approach [1]. Schema mapping (also referred to as semantic mapping) is the process of relating the attributes of source schemas to the mediated schema (sometimes using expressions in a mapping language). The output of schema matching is used as input to schema mapping algorithms [1].

Despite recent developments, setting up a full data integration system with a manually designed mediated schema requires significant human effort (e.g. domain experts and database designers). On the other hand, there are many application contexts, e.g. web, scientific data management, and personal information 
management, which do not require full integration to provide useful services [2]. These applications need to start with a data integration application in a complete automatic setting for reducing human effort and development time and to put more effort later on as needed to improving it. This setting is referred to by pay-as-you-go data integration.

The goal of our research is to study how advanced of a starting point we can build a pay-as-you-go data integration system in a fully automated setting. For building such a system, we take advantage of the background knowledge which is implied in the functional dependencies (FDs) defined on the schemas. Since probabilistic data models have shown to be promising [3-5], we build our approach on a probabilistic data model to capture the uncertainty which arises during the schema matching process. Therefore, we generate a set of Probabilistic Mediated Schemas (PMSs). The idea behind PMSs is to have several mediated schemas, each one with a probability that indicates the closeness of the corresponding mediated schema to the ideal mediated schema.

In the database literature, the closest related work to ours is that of Sarma et al. [4] which based on PMSs proposed UDI (Uncertain Data Integration), an uncertain data integration system. However, UDI may fail to capture some important attribute correlations, and thereby produce low quality answers. Let us clarify this by an example which is the same as the running example in [4].

Example 1 Consider the following schemas both describing people:

$S_{1}(\overline{\text { name, } h \text { Phone, } h A d d r, o P h o n e, o A d d r})$

$S_{2}$ (name, phone, address)

In $S_{2}$, the attribute phone can either be a home phone number or an office phone number, and the attribute address can either be a home address or an office address.

A high quality data integration system should capture the correlation between $h$ Phone and $h A d d r$ and also between oPhone and $o A d d r$. Specifically, it must generate schemas which group the address and $h A d d r$ together if phone and $h$ Phone are grouped together. Similarly it should group the address and $o A d d r$ together if phone and $o$ Phone are grouped together. In other words either of the following schemas should be generated (we abbreviate hPbone, oPhone, $h A d d r, o A d d r$ as $h P, o P, h A$, and $o A$ respectively):

$M_{1}(\{$ name, name $\},\{$ phone,$h P\},\{o P\},\{$ address, $h A\},\{o A\})$

$M_{2}(\{$ name, name $\},\{$ phone,$o P\},\{h P\},\{$ address, $o A\},\{h A\})$

UDI does not consider attribute correlations. Thus, although $M_{1}$ and $M_{2}$ may be generated by UDI, they are overwhelmed by schemas in which the attribute correlations are not respected. As a results, by producing a large number of schemas which can easily be exponential, the desirable schemas get a very low probability.

Most attribute correlations are expressed within FDs. For example let $F_{1}$ and $F_{2}$ be the set of FDs of $S_{1}$ and $S_{2}$ respectively:

$F_{1}=\{h$ Phone $\rightarrow$ hAddr, oPhone $\rightarrow$ oAddr $\}$

$F_{2}=\{$ phone $\rightarrow$ address $\}$ 
FDs in $F_{1}$ and $F_{2}$ show the correlation between the attributes in $S_{1}$ and $S_{2}$, respectively. For example, $h$ Phone $\rightarrow h A d d r$ indicates that the two attributes $h P h o n e$ and $h A d d r$ are correlated. Considering the pairs of FDs from different sources can help us extracting these correlations and achieving the goal of generating mediated schemas that represent these correlations. For example, the FD pair phone $\rightarrow$ address and $h$ Phone $\rightarrow h A d d r$ indicates that if we group phone and $h P h o n e$ together, we should also group address and $h A d d r$ together, as well as oPhone and $o A d d r$.

The specific contributions of this paper are the following.

- We propose a data integration system that takes into account attribute correlations by using functional dependencies, and captures uncertainty in mediated schemas using a probabilistic data model. Our system allows integrating a given set of data sources, as well as incrementally integrating additional sources, without needing to restart the process from scratch.

- We model the schema matching problem as a clustering problem with constraints. This allows us to generate mediated schemas using algorithms designed for the latter problem. In our approach, we build a custom distance function for representing the knowledge of attribute semantics which we extract from FDs.

- To validate our approach, we implemented it as well as baseline solutions. The performance evaluation results show significant performance gains of our approach in terms of recall and precision compared to the baseline approaches. They confirm the importance of FDs in improving the quality of uncertain mediated schemas.

The paper is organized as follows. In Section 2, we make our assumptions precise and define the problem. In Section 3, we briefly describe the architecture of our system. In Section 4, we propose our approach for schema matching. We also analyze the execution cost of our proposed approach. Section 5 describes our performance validation. Section 6 discusses related work, and Section 7 concludes.

\section{Preliminaries}

In this section, we first make precise our assumptions and give some background about PMSs. Then, we define the problem we address in this paper.

We assume that the functional dependencies between the attributes of sources are available. This is a reasonable assumption in the applications which we consider, in particular scientific applications, because the data source providers are willing to provide the full database design information, including functional dependencies. However, there are contexts such as the web in which functional dependencies are not available. For these applications, we can use one of the existing solutions, e.g. $[6,7]$ to derive functional dependencies from data. Another assumption, which we make for ease of presentation, is that the data model is relational. 
Let us formally define some basic concepts, e.g. functional dependencies and mediated schemas, and then state the problem addressed in this paper. Let $S$ be a set of source schemas, say $S=\left\{S_{1}, \ldots, S_{n}\right\}$, where for each $S_{i}, i \in[1, n], S_{i}=$ $\left\{a_{i, 1}, \ldots, a_{i, l_{i}}\right\}$, such that $a_{i, 1}, \ldots, a_{i, l_{i}}$ are the attributes of $S_{i}$. We denote the set of attributes in $S_{i}$ by $\operatorname{att}\left(S_{i}\right)$, and the set of all source attributes as $A$. That is $A=\cup_{i} \operatorname{att}\left(S_{i}\right)$. For simplicity, we assume that $S_{i}$ contains a single table. Let $F$ be the set of functional dependencies of all source schemas, say $F=\left\{F_{1}, \ldots, F_{n}\right\}$. For each $S_{i}, i \in[1, n]$, let $F_{i}$ be the set of functional dependencies among the attributes of $S_{i}$, i.e. $\operatorname{att}\left(S_{i}\right)$, where each $f d_{j}, f d_{j} \in F_{i}$ is of the form $L_{j} \rightarrow R_{j}$ and $L_{j} \subseteq \operatorname{att}\left(S_{i}\right), R_{j} \subseteq \operatorname{att}\left(S_{i}\right)$. In every $F_{i}$, there is one fd of the form $L_{p} \rightarrow R_{p}$, where $R_{p}=\operatorname{att}\left(S_{i}\right)$, i.e. $L_{p}$ is the primary key of $S_{i}$.

We assume one-to-one mappings of source attributes, meaning that each attribute can be matched with at most one attribute. We do this for simplicity and also because this kind of mapping is more common in practice. For a set of sources $\mathrm{S}$, we denote by $M=\left\{A_{1}, \ldots, A_{m}\right\}$ a mediated schema, where $A_{i} \subseteq A$, and for each $i, j \in[1, m], i \neq j \Rightarrow A_{i} \cap A_{j}=\emptyset$. Each attribute involved in $A_{i}$ is called a mediated attribute. Every mediated attribute ideally consists of source attributes with the same semantics.

Let us formally define the concept of probabilistic mediated schema (PMS). A $P M S$ for a set $S$ of source schemas is the set $N=\left\{\left(M_{1}, P\left(M_{1}\right)\right), \ldots,\left(M_{k}, P\left(M_{k}\right)\right)\right\}$ where $M_{i}, i \in[1, k]$, is a mediated schema, and $P\left(M_{i}\right)$ is its probability. For each $i, j \in[1, k], i \neq j \Rightarrow M_{i} \neq M_{j}$, i.e. $M_{i}$ and $M_{j}$ are different clusterings of $\operatorname{att}(S)$; and $\sum_{i=1}^{k} P\left(M_{i}\right) \leq 1$.

We use the precision, recall, and F-measure of the clustering for measuring the quality of the generated mediated schemas.

Now, we formally define the problem we address. Suppose we are given a set of source schemas $S$, and a set of functional dependencies $F$ and a positive integer number $k$ as input. Our problem is to efficiently find a set of $k$ probabilistic mediated schemas which have the highest F-measure.

\section{System Architecture}

The architecture of our data integration system, hereafter called IFD (Integration based on Functional Dependencies), is shown in Figure 1. IFD consists of two main parts: schema matching (part A) and query processing (part B). The components of schema matching, which operate during the set-up time of the system, are as follows:

- Attribute similarity computing: this component computes the attribute name similarity between every two source attributes.

- FD derivation: this component derives functional dependencies from data, which is an optional component of the system and is only used in the cases where functional dependencies are not given to the system.

- Distance assignment: this component uses attribute pairs similarity and functional dependencies for generating the distance function. 
- Schema matching: this component uses the distance function for generating a set of probabilistic mediated schemas.

- Single schema building: this component generates one mediated schema for the user by using the generated probabilistic mediated schemas.

The components of the query processing part is depicted in Part B of Figure 1. We include these components in the architecture of our system to provide a complete picture of a data integration system but our focus is on the schema matching part (part A). The components of part B which operate at query evaluation time are as follows:

- Query reformulation: This component uses the probabilistic mediated schemas to reformulate the user query posed against the mediated schema to a set of queries posed over the data sources.

- Query result aggregation: This component combines the results of reformulated queries and assigns a probability to every tuple in the result, based on both the probabilities of the mediated schemas and the dependency among data sources.

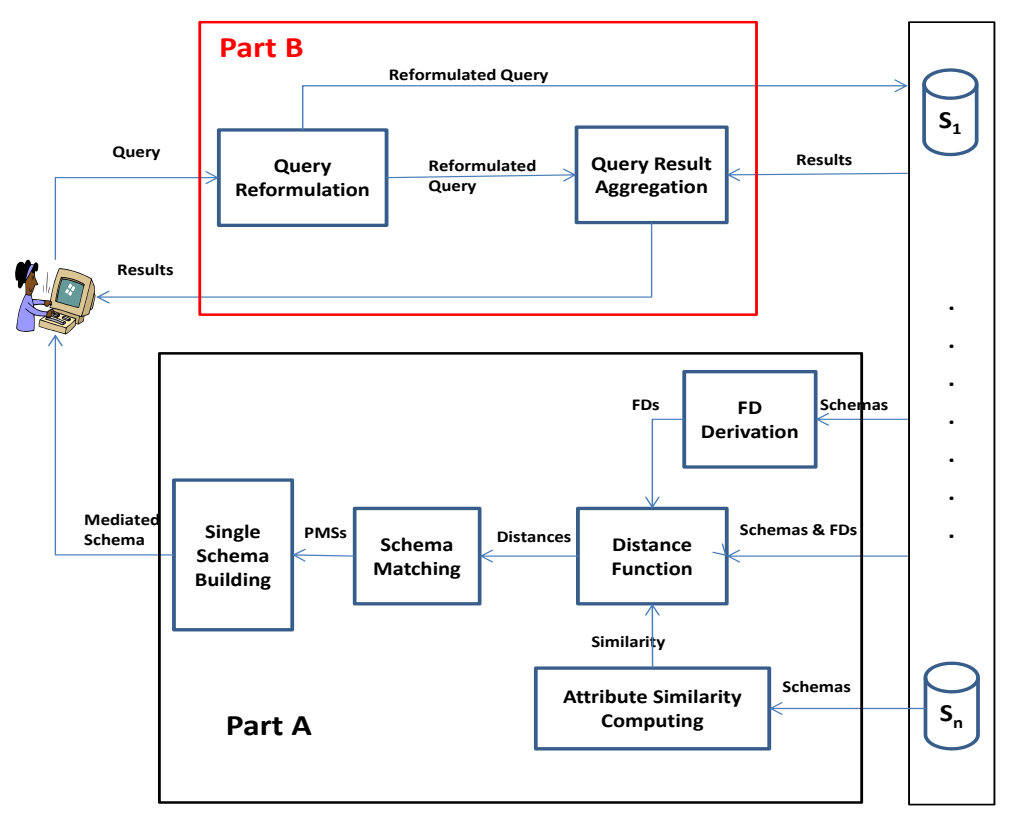

Fig. 1. IFD architecture 


\section{Schema Matching}

In this section, we present the schema matching part of IFD. To match the schemas automatically, we cluster the source attributes by putting semantically equivalent attributes in the same cluster. We use a clustering algorithm that works based on a distance function, which determines the distance between every two attributes. Specifically we use the single-link CAHC (Constrained Agglomerative Hierarchical Clustering) algorithm [8]. In the rest of this section, we first describe our distance function. Then, we describe our approach for schema matching. We then describe a useful feature of our approach. We also analyze the execution cost of the proposed algorithms. Finally, We briefly describe how the result of schema matching is used to generate the schema mappings.

\subsection{Distance Function}

Our schema matching algorithm uses a distance function for determining the distance between source attributes. To assign the distances between the attributes, we use the attributes' name similarity as well as some heuristics we introduce about FDs. In the rest of this section, we first describe our heuristics and then present the distance function algorithm.

FD Heuristics We use heuristic rules related to FDs in order to assign the distance of attributes. Before describing our heuristics, let us first define Match and Unmatch concepts. Consider $a_{1}$ and $a_{2}$ as two typical attributes. If we want to increase their chance of being put in the same cluster, we set their distance to $M D$ (i.e. Match Distance) which is 0 or a number very close to 0 . In this case, we say that we matched $a_{1}$ with $a_{2}$, and we show this by $\operatorname{Match}\left(a_{1}, a_{2}\right)$. In contrast, if we want to decrease their chance of being put in the same cluster, then we set their distance to $U M D$ (i.e. Un-Match Distance) which is 1 or a number very close to 1 . In this case, we say that we unmatched $a_{1}$ and $a_{2}$ and we show this by Unmatch $\left(a_{1}, a_{2}\right)$. Now, Let us use the following example to illustrate the heuristics.

Example 2 Consider two source schemas, both describing bibliographical information of scientific papers. In this example, primary keys are underlined; $F_{1}$ and $F_{2}$ are the sets of FDs of $S_{1}$ and $S_{2}$ respectively:

$S_{1}$ (author, title, year, institution, journal, issn)

$S_{2}$ (name, paper_title, date, af filiation, journal, serial_no, volume, issue)

$F_{1}=\{$ author $\rightarrow$ institution, journal $\rightarrow$ issn $\}$

$F_{2}=\{$ name $\rightarrow$ affiliation, journal $\rightarrow$ serial_no $\}$

Heuristic 1 Let $S_{p}$ and $S_{q}, p \neq q$, be two source schemas. Then,

$$
\begin{gathered}
\operatorname{Match}\left(a_{p, i}, a_{q, k}\right) \Rightarrow \operatorname{unmatch}\left(a_{p, i}, a_{q, l}\right) \wedge \operatorname{unmatch}\left(a_{q, k}, a_{p, j}\right) \\
\text { where } a_{p, i} \in \operatorname{att}\left(S_{p}\right), a_{p, j} \in \operatorname{att}\left(S_{p}\right) \backslash\left\{a_{p, i}\right\}, a_{q, k} \in \operatorname{att}\left(S_{q}\right), a_{q, l} \in \operatorname{att}\left(S_{q}\right) \backslash\left\{a_{q, k}\right\} .
\end{gathered}
$$


Intuitively, this heuristic means that each attribute can be matched with at most one attribute of the other source.

Heuristic 2 Let $f d_{p}: a_{p, i} \rightarrow a_{p, j}$ and $f d_{q}: a_{q, k} \rightarrow a_{q, l}$ be two FDs, where $f d_{p} \in F_{p}, f d_{q} \in F_{q}, p \neq q$. Then, similarity $\left(a_{p, i}, a_{q, k}\right)>t_{L} \Rightarrow \operatorname{Match}\left(a_{p, j}, a_{q, l}\right)$ where $t_{L}$ is a certain threshold and similarity is a given similarity function.

In this heuristic, We consider the set of facts that the two sources are assumed to be from the same domain, and both attributes $a_{p, j}$ and $a_{q, l}$ are functionally determined by the attributes $a_{p, i}$, and $a_{q, k}$ respectively, which themselves have close name similarity. Thus, we heuristically agree that: the probability of $\operatorname{Match}\left(a_{p, j}, a_{q, l}\right)$ is higher than that of $\operatorname{Match}\left(a_{p, j}, a_{q, s}\right)$ and $\operatorname{Match}\left(a_{q, l}, a_{p, r}\right)$, where $a_{q, s} \in \operatorname{att}\left(S_{q}\right) \backslash\left\{a_{q, l}\right\}$ and $a_{p, r} \in S_{p} \backslash\left\{a_{p, j}\right\}$. Therefore, in such a case we match $a_{p, j}$ with $a_{q, l}$ to reflect this fact. Note that this heuristic has a general form in which there are more than one attribute on the sides of the FDs (see Section 4.1).

Let us apply heuristic 2 on Example 2. We have the FD journal $\rightarrow$ issn from $S_{1}$, and journal $\rightarrow$ serial_no from $S_{2}$. There is only one attribute at the left side of these FDs, and their name similarity is equal to 1 that is the maximum similarity value. Thus, we match the issn with the serial_no which appear on the right side of these FDs. Notice that approaches which only rely on name similarity, probably match issn with issue, which is a wrong decision.

Heuristic 3 Let $P K_{p}$ and $P K_{q}, p \neq q$, be the primary keys of $S_{p}$ and $S_{q}$ respectively. Then,

$$
\begin{gathered}
\left(\exists a_{p, i} \in P K_{p}, a_{q, j} \in P K_{q} \mid\left(a_{p, i}, a_{q, j}\right)=\underset{a_{p} \in P K_{p}, a_{q} \in P K_{q}}{\arg \max } \operatorname{similarity}\left(a_{p}, a_{q}\right)\right) \wedge \\
\left(\operatorname{similarity}\left(a_{p, i}, a_{q, j}\right)>t_{P K}\right) \Rightarrow \operatorname{Match}\left(a_{p, i}, a_{q, j}\right)
\end{gathered}
$$

where $t_{P K}$ is a certain threshold and similarity is a given similarity function.

Let us explain the idea behind heuristic 3. Since we assume sources are from the same domain, there are a number of specific attributes which can be part of the primary key. Although these attributes may have different names in different sources, it is reasonable to expect that some of these attributes from different sources can be matched together. Obviously, we can set $t_{P K}$ to a value less than the value we set for $t_{L}$ because typically the probability of finding matching attributes in the primary key attributes is higher than the other attributes. After matching $a_{p, i}$ with $a_{q, j}$, we remove them from $P K_{p}$ and $P K_{q}$ respectively, and continue this process until the similarity of the pair with the maximum similarity is less than the threshold $t_{P K}$ or one of the $P K_{p}$ or $P K_{q}$ has no more attributes to match.

Coming back to Example 2, it is reasonable to match the attributes: author, title, and year of $S_{1}$ with name, paper_title, and date of $S_{2}$ rather than with other attributes of $S_{2}$, and vice versa. The attribute pair with the maximum similarity is (title,paper_title). If we choose a good threshold, we can match 
these attributes together. The similarity of other attribute pairs is not high enough to pass the wisely selected threshold values.

Heuristic 4 Let $P K_{p}$ and $P K_{q}, p \neq q$, be the primary keys of $S_{p}$ and $S_{q}$ respectively. Then,

$$
\begin{aligned}
&\left(\exists a_{p, i} \in P K_{p}, a_{q, j}\right. \in P K_{q}, f d_{p} \in F_{p}, f d_{q} \in F_{q} \mid \\
&\left.f d_{p}: a_{p, i} \rightarrow R_{p}, f d_{q}: a_{q, j} \rightarrow R_{q}\right) \Rightarrow \operatorname{Match}\left(a_{p, i}, a_{q, j}\right)
\end{aligned}
$$

Heuristic $5\left(R H S(1) \wedge R_{p}=\left\{a_{p, r}\right\} \wedge R_{q}=\left\{a_{q, s}\right\}\right) \Rightarrow \operatorname{Match}\left(a_{p, r}, a_{q, s}\right)$

Heuristic 4 is applicable when we have two attributes in two primary keys which each of them is the single attribute appearing at the left side of a FD. In this case, we match these attributes with each other. We also match the attributes on the right sides of the two FDs if there is only one attribute appearing at the right side of them (heuristic 5).

Using heuristic 4 for Example 2, we match author with name which is a right decision. We do this because of the two FDs: author $\rightarrow$ institution and name $\rightarrow$ affiliation. We also match institution with af filiation which are the only attributes appearing at the right side of these FDs based on heuristic 5 .

Heuristic 6 Let $P K_{p}$ and $P K_{q}, p \neq q$, be the primary keys of $S_{p}$ and $S_{q}$ respectively. Then,

$$
\begin{gathered}
\left(\forall a_{p, r} \in P K_{p} \backslash\left\{a_{p, i}\right\}, \exists a_{q, s} \in P K_{q} \backslash\left\{a_{q, j}\right\} \mid \operatorname{Match}\left(a_{p, r}, a_{q, s}\right)\right) \wedge \\
\left(\left|P K_{p}\right|=\left|P K_{q}\right|\right) \Rightarrow \operatorname{Math}\left(a_{p, i}, a_{q, j}\right)
\end{gathered}
$$

Heuristic 6 is applicable when all attributes of $P K_{p}$ and $P K_{q}$ have been matched, and only one attribute is left in each of them. In such case we match these two attributes with each other hoping that they are semantically the same. Coming back to Example 2, there is only one attribute left in each of the primary keys that we have not yet matched (i.e. year, date) that we can match using this heuristic.

Distance Function Algorithm Algorithm 1 describes how we combine the attributes' name similarity and FD heuristics to build the distance function. Steps 2-10 of the algorithm apply heuristic 2. They look for FD pairs from different sources which their left sides match together and then try to match attribute pairs on the right sides of these FDs. After finding such FDs, steps 5-10 repeatedly find the attribute pairs $\left(a_{p}, a_{q}\right)$ whose similarity is maximum. If the similarity of $a_{p}$ and $a_{q}$ is more than threshold $t_{R}$, their distance is set to $M D$ (Match Distance), and the distances between each of them and any other source-mates are set to UMD (Unmatch Distance). The algorithm uses the DoMatch procedure for matching and unmatching attributes. It gets the attributes which should be matched as parameter, matches them, and unmatches every one of them with the other ones' source-mates. Generally, whenever the 


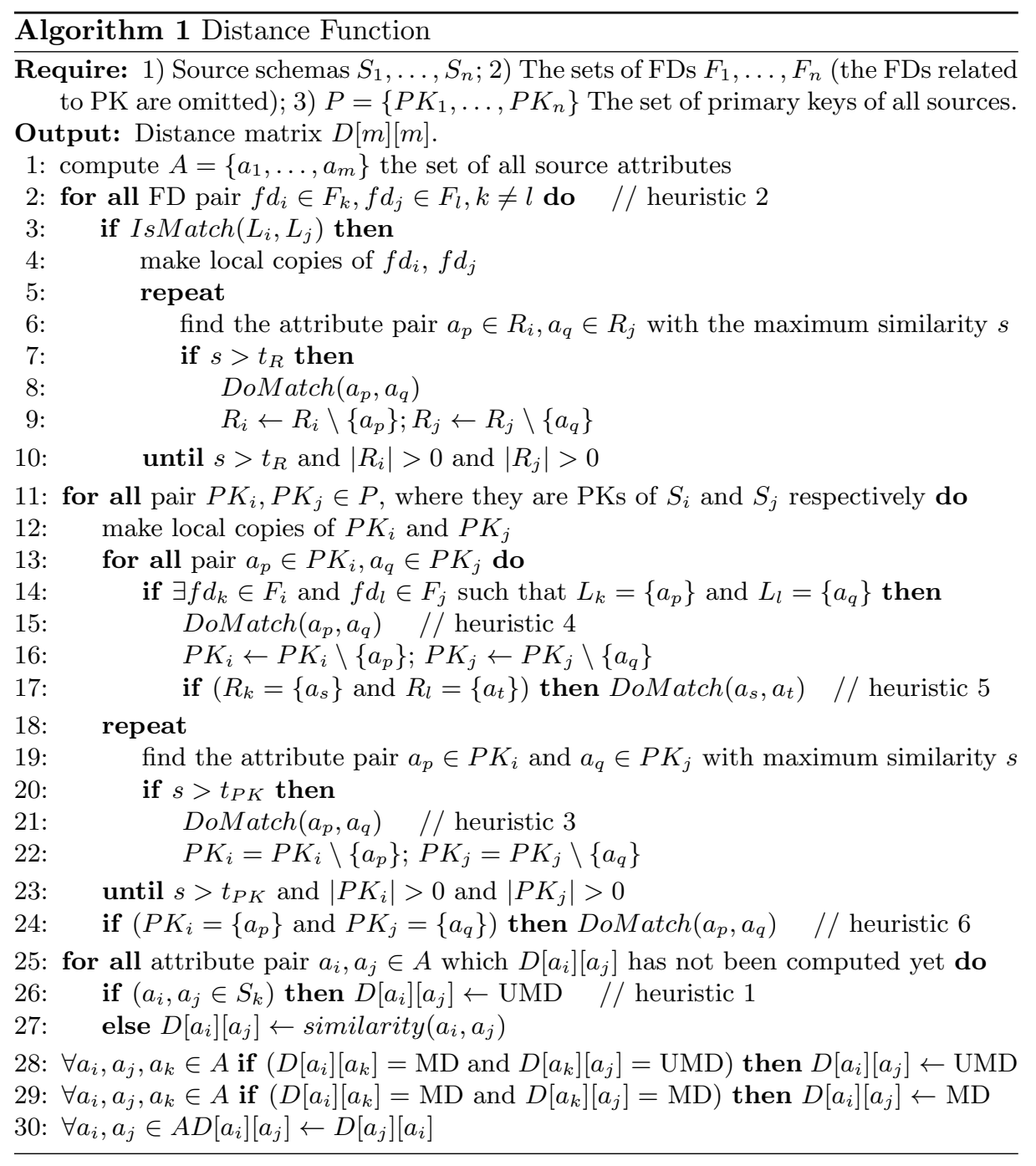

algorithm matches two attributes with each other, it also unmatches the two of them with the other one's source-mates because every attribute of a source can be matched with at most one attribute of every other source. Steps 9 remove the matched attributes from the list of unmatched attributes.

The IsMatch function, which is used by step 3, takes as parameter the left sides of two FDs and returns true if they can be matched together, otherwise it returns false. It first checks whether the input parameters are two sets of the same size. Then, it finds the attribute pair with maximum name similarity and treats it as matched pair by removing the attributes from the list of unmatched attributes if their similarity is more than threshold $t_{L}$. It repeats the matching process until there is no more attribute eligible for matching. After the matching 
loop is over, the function returns true if all attribute pairs have been matched together, otherwise it returns false which means the matching process has not been successful. Notice that we do not reflect the matching of attributes of the left sides of FDs in the distance matrix. The reason is that for these attributes (in contrast to those on the right side), the matching is done just based on attribute name similarity and not the knowledge in FDs.

Please notice that we use three different similarity thresholds (i.e. $t_{L}, t_{R}$, and $\left.t_{P K}\right)$ to have more flexibility in the matching. However, we need to set them carefully. If we set them to high values, we prevent wrong matching but may miss some pairs that should have been matched. On the other hand, if we set thresholds to low values, we increase the number of correctly matched pairs but also increase the number of wrongly matched pairs. In other words, setting the threshold values is a trade off between precision and recall. Aside from this, the inequality between them is important as we explain below. We know that $t_{L}$ is the similarity threshold for matching attributes at the left sides of FDs. Since the matching of left sides of FDs is taken as evidence for matching the right sides of them, $t_{L}$ needs to be chosen carefully. Setting it to low values, results in wrong matchings. On the other hand, we use $t_{R}$ as similarity threshold for matching attributes on the right sides of FDs. Since we already have evidence for matching them, we can be more relaxed in setting $t_{R}$ by setting it to values lower than $t_{L}$. The same argument goes for the value of $t_{P K} \cdot t_{P K}$ is the similarity threshold for matching PK attributes. Since these attributes are a subset of source attributes, it is reasonable to set $t_{P K}$ to lower values than $t_{L}$ and $t_{R}$.

In steps 11-24, we apply heuristics 3,4, 5 and 6. Steps 13-17 check every attribute pair of two PKs to see if they are the only attributes at the left sides of two FDs. If yes, then these attributes are matched together. Steps 18-23 find the attribute pair with the maximum name similarity and if it is more than threshold $t_{P K}$, the attributes are matched together. The matching process continues until there is at least one attribute in every PK and the similarity of the attribute pair with the maximum similarity is more than threshold $t_{P K}$. If each of the two PKs has only one attribute left, step 24 matches them together based on heuristic 6 .

In steps 25-27, we set the distances of attribute pairs which have not been set yet. Step 26 applies heuristic1 by setting the distance of the attributes from the same source to $U M D$. The distance of other attribute pairs is set to their name similarity. Steps 28-29 perform a transitive closure over the match and unmatch constraints. Step 30 deals with the symmetric property of the distance function to ensure that the returned distance is independent from the order of attributes.

The matching and unmatching decisions made by a distance function should be consistent with each other. More precisely, a consistent distance function should not satisfy the following condition:

$$
\exists a_{i}, a_{j}, a_{k} \in A \mid \operatorname{match}\left(a_{i}, a_{j}\right) \wedge \operatorname{match}\left(a_{j}, a_{k}\right) \wedge \operatorname{unmatch}\left(a_{i}, a_{k}\right) .
$$

The following proposition shows that our distance function is consistent.

Proposition 1. Algorithm 1 returns a consistent distance function. 
Proof. We first show that if inconsistency exists, it is removed by step 32 of the algorithm, i.e. the first transitive closure rule. Then, we show that order of applying the transitive closure rules in Algorithm 1 is the only correct order. Let us prove the first part. Suppose steps 1-31 of the algorithm create an inconsistency so that condition (2) satisfies. Then, as the result of step 32 of the algorithm, either match $\left(a_{i}, a_{j}\right)$ changes to unmatch $\left(a_{i}, a_{j}\right)$ or match $\left(a_{j}, a_{k}\right)$ changes to unmatch $\left(a_{j}, a_{k}\right)$. It is clear that the inconsistency between $a_{i}, a_{j}$, and $a_{k}$ is removed with either of the changes. Without the loss of generality, we assume that $\operatorname{match}\left(a_{i}, a_{j}\right)$ changes to unmatch $\left(a_{i}, a_{j}\right)$. Then, if there exists $a_{l} \in A$, so that condition (2) satisfies for $a_{i}, a_{j}$, and $a_{l}$ as a result of the change, step 32 removes it too. Thus, step 32 removes all of the inconsistencies in the cost of losing possibly correct match pairs.

Let us prove the second part. Suppose that steps 1-31 of the algorithm create an inconsistency so that condition (2) satisfies and we change the order of transitive closure rules. By first applying rule 2 , unmatch $\left(a_{i}, a_{k}\right)$ changes to $\operatorname{match}\left(a_{i}, a_{k}\right)$. However, we already unmatched $a_{i}$ with $a_{k}$ as the result of matching $a_{i}$ with one of the source-mates of $a_{k}$, say $a_{l}$. Thus, we have: $\operatorname{match}\left(a_{k}, a_{i}\right)$ and match $\left(a_{i}, a_{l}\right)$, which results in match $\left(a_{k}, a_{l}\right)$ by applying rule 2 to them. This means that we matched two attributes $a_{k}$ and $a_{l}$ from the same source. Thus, changing the order of transitive closure rules does not remove the inconsistency but propagates it.

\subsection{Schema Matching Algorithm}

We now describe the schema matching algorithm which works based on the CAHC clustering method. This algorithm takes as input the source schemas, distance function which is computed using Algorithm 1, and the needed number of mediated schemas $(\mathrm{k})$ which is specified by the user, and returns a set of probabilistic mediated schemas to the user. The algorithm stores all created mediated schemas in the set M, which is initialized to $\emptyset$. The schema matching algorithm works as follows:

1. Put each attribute of the source schemas in one cluster, and add the generated mediated schema to the set M.

2. Find the two clusters with the minimum distance while the distance between two clusters is defined as follows: if the clusters have two attributes from the same source, the distance between them is infinity; otherwise the minimum distance between two attributes, each from one of the two clusters, is regarded as the distance between the two clusters.

3. If the minimum distance, found in 2 , is not equal to infinity, then merge these clusters; add the newly created mediated schema to M; and go to 2 . Otherwise, go to 4.

4. For each mediated schema in M, compute FD-point as the number of match decisions that are respected by the mediated schema. Notice that by match decision, we mean the attribute pair whose returned distance by the distance function is MD. 
5. If $|M|>k$, then select $\mathrm{k}$ mediated schemas of $\mathrm{M}$ with the highest FDpoint; assign probability $\frac{F D \text { point }_{i}}{\sum_{i=1}^{k} F \text { Dpoint }}$ to each selected mediated schema; and return them. Otherwise, assign probability $\frac{1}{|M|}$ to each mediated schema in $\mathrm{M}$, and return them. Notice that in case of ties, we randomly select mediated schemas.

\subsection{Adding Data Sources Incrementally}

IFD starts with a given set of sources and ends up generating several mediated schemas from these sources. A useful property of IFD is that it allows new sources to be added to the system on the fly. Let $S_{n+1}$ be the source which we want to add. By comparing $S_{n+1}$ with each $S_{i}, i \in[1 . . n]$, we can compute the distance between every attribute of $S_{n+1}$ and every attribute of $S_{i}$ in the same way that we did for computing the distances between the attributes of $S_{1} . . S_{n}$. After computing the distances, we consider every PMS, say $M_{j}, j \in[1 . . k]$ and for every attribute $a_{p} \in S_{n+1}$, we find the closest attribute $a_{q} \in A$ and put $a_{p}$ in the same cluster as that of $a_{q}$. We repeat this process for every PMS.

This is a useful property of IFD which is needed in the contexts which we do not have all sources at hand when we start setting up the data integration application and we need to add them incrementally when they become available.

\subsection{Execution Cost Analysis}

In this section, we study the execution costs of our schema matching and distance function algorithms.

Theorem 1. Let $m$ be the number of the attributes of all sources, then the running time of Algorithm 1 and the schema matching algorithm together is $\theta\left(m^{3}\right)$.

Proof. The basis for our schema matching algorithm is the single-link CAHC (Constrained Agglomerative Hierarchical Clustering) algorithm in which the number of clusters is determined by the arity of the source with the maximum arity. Let $\mathrm{m}$ be the number of the attributes of all sources. The time complexity of the single-link AHC algorithm implemented using next-best-merge array $(\mathrm{NBM})$ is $\Theta\left(m^{2}\right)[9]$.

Let us now analyze the running time of the distance function algorithm. Most of the algorithm is devoted to matching left and right sides of FDs, or the attributes of PKs. Let $\mathrm{c}$ be the arity of the source with the maximum arity, and $\mathrm{f}$ the maximum number of FDs that a source may have, which is a constant. The number of attributes on the left and right side of a FD is at most equal to the arity of its source, so its upper bound is $c$. Thus, matching both sides of two FDs takes $\Theta\left(c^{2}\right)$ time which is equal to $\Theta(1)$ because $c$ is a constant. This argument also holds for matching PKs' attributes because the algorithm only checks the FDs of the two sources (which each one at most has f FDs), not the FDs of all sources. 
Let $n$ be the number of sources, then we have at most $f \times n$ FDs. The algorithm checks every FD pair for matching. Thus, it takes $\frac{f \times n \times(f \times n-1)}{2} \times \Theta(1)$ time for matching FDs which is equal to $\left(n^{2} \times f^{2}\right)$. By taking $f$, i.e. the maximum number of FDs, as a constant, the complexity is $\Theta\left(n^{2}\right)$. In the same way, the time complexity for matching PKs is $\Theta\left(n^{2}\right)$.

The transitive closure part of the algorithm is done in $\theta\left(\mathrm{m}^{3}\right)$ time, where $m$ is the total number of attributes. The last part of the algorithm that guarantees symmetric property takes $\theta\left(\mathrm{m}^{3}\right)$. Since the number of attributes is at least the number of sources, we have $m \geq n$. Thus, the transitive closure of attributes dominates all other parts of the algorithm and the running time of the algorithm is $\theta\left(\mathrm{m}^{3}\right)$. As a result, the running time of the schema matching and the distance function algorithms together is $\theta\left(\mathrm{m}^{3}\right)$.

\subsection{Schema Mapping}

The result of schema matching is fed to the schema mapping algorithm for generating the mappings which are used in query processing. In our data integration solution, we assume that every attribute in the data sources can be matched with at most one attribute in other data sources which means we only consider one-toone mappings. We do this for simplicity and also because this kind of mapping is more common in practice. We also assume single-table data sources. These two assumptions greatly simplify the schema mapping algorithm. For generating the mappings, we can rely on PMSs which implicitly contain the mappings for answering user queries. We do not provide the details of the schema mapping algorithm here due to its simplicity and also lack of space.

\section{$5 \quad$ Experiments}

We now present our experimental results. The main goal of our experiments is to show the effect of using functional dependencies on the quality of generated mediated schemas. To examine the contribution of using a probabilistic approach, we compare our approach with two traditional baseline approaches that do not use probabilistic techniques, i.e. they generate only one single deterministic mediated schema. We also compare our system with the one presented in [4] which is the closest to ours.

We implemented IFD in Java, and we used Weka 3-7-3 classes for implementing the hierarchical clustering component. We used the SecondString tool ${ }^{1}$ to compute the Jaro Winkler similarity [10] of attribute names in pair-wise attribute comparison. We conducted our experiments on a Windows XP machine with Intel core $2 \mathrm{GHz}$ CPU and 2GB memory.

The parameters we used in our experiments are listed in Table 1 . We set the number of reuired mediated schemas (denoted as $k$ ) to 1000 , which is relatively high, in order to return all eligible mediated schemas. Our experiments showed similar results when we varied $k$ considerably (e.g. $k=5$ ).

\footnotetext{
${ }^{1}$ Secondstring. http://secondstring. sourceforge.net/
} 
Table 1. Parameters

\begin{tabular}{lr}
\hline Parameter & Values \\
\hline$k:$ the number of required mediated schemas & 1000 \\
$t_{P K}:$ similarity threshold for PK attributes & 0.7 \\
$t_{L}:$ similarity threshold for attributes on the left side of FDs & 0.9 \\
$t_{R}:$ similarity threshold for attributes on the right side of FDs & 0.8 \\
MD: match distance & 0 \\
UMD: unmatch distance & 1 \\
\hline
\end{tabular}

For evaluating our system, we used a dataset which we designed manually. This dataset ${ }^{2}$ consists of 17 single-table schemas in the university domain. For having variety in attribute names, we used Google Search with "computer science" and "course schedule" keywords and picked up the first 17 related results. For every selected webpage, we designed a single-table schema which could be the data source of the course schedule information on that webpage and we used data labels as attribute names of the schema. Also, we created primary key and functional dependencies for every schema using our knowledge of the domain.

We tested the generated mediated schemas against the ideal mediated schema, which we created manually, to measure their quality.

We tested the generated mediated schemas against the ideal mediated schema, which we created manually, to measure their quality. Since each mediated schema corresponds to a clustering of source attributes, we measured its quality by computing the precision, recall, and F-measure of the clustering. Let TP (True Positive) be the number of attribute pairs that are clustered correctly, FP (False Positive) be the number of attribute pairs that are clustered wrongly, and $F N$ (False Negative) be the number of attribute pairs which are clustered together in the manually created schema but such pairs do not exist in the aforementioned schema. The three metrics are defined as follows: Precision: $P=\frac{T P}{T P+F P}$; Recall: $R=\frac{T P}{T P+F N}$; F-measure: $F=\frac{2 \times P \times R}{P+R}$. We computed the metrics for each individual mediated schema, and summed the results weighted by their respective probabilities.

As far as the authors know, the most competing approach to ours (IFD) is that of Sarma et al. [4] which we denote by UDI as they did. Thus, we compare our solution with UDI as the most competing probabilistic approach. We implemented UDI in Java. We used the same tool in our approach for computing pair-wise attribute similarity as in UDI. Also, we set the parameters edge-weight threshold and error bar to 0.85 and 0.02 respectively. Since the time complexity of UDI approach is exponential to the number of uncertain edges, we selected the above values carefully to let it run.

\footnotetext{
$\overline{2}$ The dataset is available at http://www.science.uva.nl/CO-IM/papers/IFD/ IFD-test-dataset.zip
} 


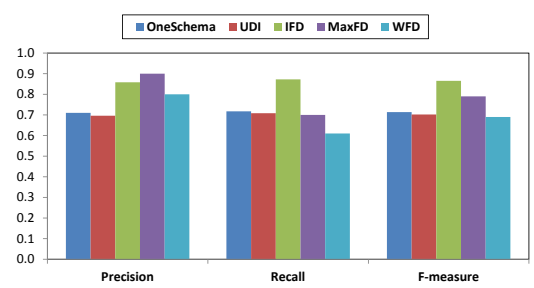

Fig. 2. Performance comparison of IFD with competing approaches

We considered two baseline approaches that create a single mediated schema, to evaluate the performance gain of using a probabilistic technique. These approaches are as follows:

- MaxFD: creates a deterministic mediated schema as follows. In the schema matching algorithm in section 4.2 , we count the number of FD recommendations and obtain the maximum possible FD-point, then we stop at the first schema which gets this maximum point.

- OneSchema: creates a deterministic mediated schema based on Algorithm 4.1 in [4]. We set frequency threshold to 0 and the edge weight threshold to 0.85 .

Moreover, to examine the contribution of using functional dependencies in the quality of generated mediated schemas, we considered Algorithm 2 without taking advantage of the FD recommendations (WFD) and compared it to our approach.

In our first experiment, we compare the quality of mediated schemas generated by our approach (IFD) with the ones generated by UDI and other competing approaches. Figure 2 compares the results measuring precision, recall, and F-measure of IFD, UDI, OneSchema, MaxFD, and WFD. As this Figure shows, IFD obtains better results than UDI. IFD improves precision by $23 \%$, recall by $22 \%$, and F-measure by $23 \%$.

This Figure also shows the contribution of using FD recommendations in the quality of the results. WFD (Without FD) shows the results of our approach without using FD recommendations. It is obvious that using these recommendations has considerable effect on the results.

Moreover, Figure 2 shows the performance gain of using a probabilistic approach rather than a single deterministic schema approach. MaxFD applies all of the FD recommendations to obtain the mediated schema with the maximum FD-point, then stops and returns the resulted mediated schema. On the other hand, IFD does not stop after applying all FD recommendations but since there is no further FD recommendation, it starts merging clusters based on the similarity of their attribute pairs. This increases recall considerably, but reduces precision a little because some pairs are clustered wrongly. Overall, IFD improves F-measure by $8 \%$ compared to MaxFD. On the other hand, this Figure shows that UDI does not get such performance gain compared to OneSchema 


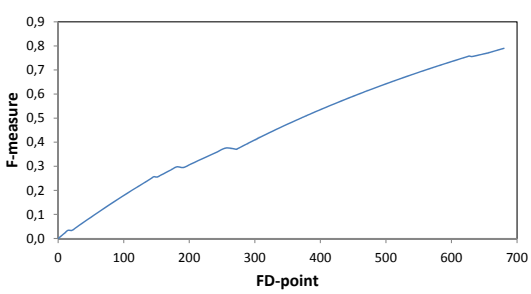

Fig. 3. F-measure vs. FD-point

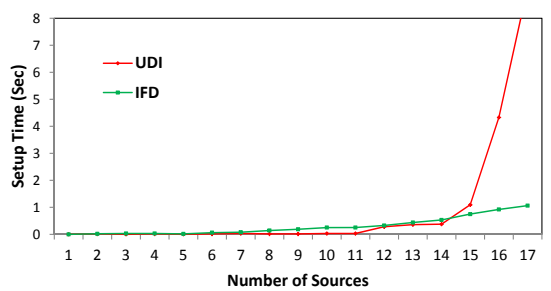

Fig. 4. Execution time of IFD and UDI

which creates a single deterministic schema. This happens because UDI cannot select the high quality schemas among the generated schemas.

In the second experiment, we study the effect of using FDs on the quality of generated mediated schemas. Figure 3 shows how F-measure increases with increasing FD-point up to 680 which is the maximum possible value in the tested dataset. The starting point is when we have one cluster for every attribute. We have not used any recommendation at this point yet; as a result, $F D$-point $=0$. Also it is clear that precision $=1$ and recall $=0$, thus $F$-measure $=0$. As we begin merging clusters using recommendations, FD-point increases and this increases the F-measure as well. The increase in FD-point continues until it reaches its maximum possible value in the tested dataset. We consider all generated mediated schemas with maximum FD-point value as schemas eligible for being in the result set.

In our final experiment, we measure the effect of the number of sources $(n)$ on IFD's execution time. By execution time, we mean the setup time needed to integrate $n$ data sources. For IFD, the execution time equals to the execution time of computing distances using Algorithm 1 plus the execution time of generating mediated schemas using Algorithm 2. For UDI, we only consider the time needed to generate mediated schemas to be fair in our comparison. For UDI, the execution time is the time needed to create the mediated schemas. Figure 4 shows how the execution times of IFD and UDI increase with increasing $n$ up to 17 (the total number of sources in the tested dataset). The impact of the number of sources on the execution time of IFD is not as high as that of UDI. While in the beginning, the execution time of UDI is a little lower than IFD, it dramatically increases eventually. This is because the execution time of IFD is cubic to the number of the attributes of sources (see Section 4.4). But, the execution time of UDI is exponential to the number of uncertain edges. This shows that IFD is much more scalable than UDI.

\section{Related Work}

A considerable amount of literature has been published on automatic schema matching during the last three decades (see [11] for a survey). They studied how to use various clues to identify the semantics of attributes and match them. An important class of approaches, which are referred to by constraint matchers, uses 
the constraints in schemas to determine the similarity of schema elements. Examples of such constraints are data types, value ranges, uniqueness, optionality, relationship types, and cardinalities. For instance, OntoMatch [12], DIKE [13], and SASMINT [14] use this type of matcher. Our approach is different, since we use an uncertain approach for modeling and generating mediated schemas. Thus, the heuristic rules we use as well as the way we decrease the distance of the attributes is completely different. In addition, we take advantage of FDs. The proposals in [15] and [16] also consider the role of FDs in schema matching. However, our heuristic rules and the way we combine it with attribute similarity is completely different with these proposals. The Similarity Flooding algorithm (SF) [17] uses a similarity graph to propagate the similarity between attributes. Our work is different from SF in the way that we do not propagate attribute similarity but instead we propagate the matching and unmatching of the attributes.

To the best of our knowledge, the closest work to ours is that of Sarma et al. [4] which we denote as UDI in this paper. UDI creates several mediated schemas with probabilities attached to them. To do so, it constructs a weighted graph of source attributes and distinguishes two types of edges: certain and uncertain. Then, a mediated schema is created for every subset of uncertain edges. Our approach has several advantages over UDI. The time complexity of UDI's algorithm for generating mediated schemas is exponential to the number of uncertain edges (i.e. attribute pairs) but that of our algorithm is PTIME (as shown in Section 4.4), therefore our approach is much more scalable. In addition, the quality of mediated schemas generated by our approach has shown to be considerably higher than that of UDI. Furthermore, the mediated schemas generated by our approach are consistent with all sources, while those of UDI may be inconsistent with some sources.

\section{Conclusion and Future Work}

In this paper, we proposed IFD, a data integration system with the objective of automatically setting up a data integration application. We established an advanced starting point for pay-as-you-go data integration systems. IFD takes advantage of the background knowledge implied in FDs for finding attribute correlations and using it for matching the source schemas and generating the mediated schema. We built IFD on a probabilistic data model in order to model the uncertainty in data integration systems.

We validated the performance of IFD through implementation. We showed that using FDs can significantly improve the quality of schema matching (by $26 \%$ ). We also showed the considerable contribution of using a probabilistic approach (10\%). Furthermore, we showed that IFD outperforms UDI, its main competitor, by $23 \%$ and has cubic scale up compared to UDI's exponential execution cost.

As future work, we plan to incorporate constraints other than PK and FDs for better specifying the distance between attributes and improve the quality of generated mediated schemas. Also, using machine learning techniques for getting 
the user feedback and improving the semantic integration of the system can be a possibility of future work. Moreover, we believe that using FDs is very promising for dealing with multi-table sources since foreign keys which are used for linking tables in relational model, are modeled using FDs. Extension of our approach to multi-table sources can be another future work direction.

\section{References}

1. Özsu, M.T., Valduriez, P.: Principles of Distributed Database Systems, 3rd Edition. Springer (2011)

2. Madhavan, J., Cohen, S., Dong, X.L., Halevy, A.Y., Jeffery, S.R., Ko, D., Yu, C.: Web-scale data integration: You can afford to pay as you go. In: Proc. of CIDR. (2007)

3. Dong, X.L., Halevy, A.Y., Yu, C.: Data integration with uncertainty. VLDB J. 18(2) (2009) 469-500

4. Sarma, A.D., Dong, X., Halevy, A.Y.: Bootstrapping pay-as-you-go data integration systems. In: Proc. of SIGMOD. (2008)

5. Akbarinia, R., Valduriez, P., Verger, G.: Efficient Evaluation of SUM Queries Over Probabilistic Data. TKDE to appear (2012)

6. Huhtala, Y., Kärkkäinen, J., Porkka, P., Toivonen, H.: Tane: An efficient algorithm for discovering functional and approximate dependencies. Comput. J. 42(2) (1999) $100-111$

7. Wang, D.Z., Dong, X.L., Sarma, A.D., Franklin, M.J., Halevy, A.Y.: Functional dependency generation and applications in pay-as-you-go data integration systems. In: Proc. of WebDB. (2009)

8. Davidson, I., Ravi, S.S.: Using instance-level constraints in agglomerative hierarchical clustering: theoretical and empirical results. Data Min. Knowl. Discov. 18(2) (2009) 257-282

9. Manning, C., Raghavan, P., Schutze, H.: Introduction to information retrieval. Volume 1. Cambridge University Press Cambridge (2008)

10. Cohen, W.W., Ravikumar, P.D., Fienberg, S.E.: A comparison of string distance metrics for name-matching tasks. In: Proc. of IIWeb. (2003)

11. Rahm, E., Bernstein, P.A.: A survey of approaches to automatic schema matching. VLDB J. 10(4) (2001) 334-350

12. Bhattacharjee, A., Jamil, H.M.: Ontomatch: A monotonically improving schema matching system for autonomous data integration. In: Proc. of Conference on Information Reuse \& Integration. (2009)

13. Palopoli, L., Terracina, G., Ursino, D.: Dike: a system supporting the semiautomatic construction of cooperative information systems from heterogeneous databases. Softw., Pract. Exper. 33(9) (2003) 847-884

14. Unal, O., Afsarmanesh, H.: Semi-automated schema integration with sasmint. Knowl. Inf. Syst. 23(1) (2010)

15. Biskup, J., Embley, D.W.: Extracting information from heterogeneous information sources using ontologically specified target views. Inf. Syst. 28(3) (2003) 169-212

16. Larson, J.A., Navathe, S.B., Elmasri, R.: A theory of attribute equivalence in databases with application to schema integration. IEEE Trans. Software Eng. 15(4) (1989) 449-463

17. Melnik, S., Garcia-Molina, H., Rahm, E.: Similarity flooding: A versatile graph matching algorithm and its application to schema matching. In: Proc. of ICDE. (2002) 